$11-1-2005$

\title{
Determination of Optimal Block Designs With Pre-assigned Variance For Elementary Contrasts
}

Seemon Thomas

St.Thomas College,Pala, ktm_stpala@sancharnet.in

Alex Thannippara

St.Thomas College, Pala, ktm_stpala@sancharnet.in

S. C. Bagui

University of West Florida, sbagui@uwf.edu

D. K. Ghosh

Saurashtra University, ghosh_dkg@rediffmail.com

Follow this and additional works at: http://digitalcommons.wayne.edu/jmasm

Part of the Applied Statistics Commons, Social and Behavioral Sciences Commons, and the Statistical Theory Commons

\section{Recommended Citation}

Thomas, Seemon; Thannippara, Alex; Bagui, S. C.; and Ghosh, D. K. (2005) "Determination of Optimal Block Designs With Preassigned Variance For Elementary Contrasts," Journal of Modern Applied Statistical Methods: Vol. 5 : Iss. 2 , Article 13. DOI: $10.22237 /$ jmasm/1162354320

Available at: http://digitalcommons.wayne.edu/jmasm/vol5/iss2/13 


\title{
Determination of Optimal Block Designs With Pre-assigned Variance For Elementary Contrasts
}

\author{
Seemon Thomas Alex Thannippara \\ St.Thomas College, Pala \\ Department of Statistics
}
S.C. Bagui
University of West Florida
D.K. Ghosh
Saurashtra University
Department of Mathematics and Statistics
Department of Mathematics Statistics

A method for obtaining optimal designs from the class of variance balanced and connected designs was developed for comparing treatment effects with a pre-assigned variance. The properties of the C-matrix of a block design are employed in developing this method. Some new results concerning the design parameters and the non-zero characteristic root of the C-matrix are also presented.

Key words: Contrasts, C-matrix, connected design, balanced incomplete block design, optimal design.

\section{Introduction}

Block designs have a wide range of applications in agriculture, engineering, medicine, social and behavioral sciences. In experimental designs for social and behavioral sciences problems, the

Seemon Thomas is Lecturer of Statistics. His areas of research are construction of optimal designs and statistical modeling. email:ktm_stpala@sancharnet.in._Alex Thannippara is Lecturer of Statistics. His areas of research are construction of combinatorial and optimal designs. email:ktm_stpala@sancharnet.in._Subhash Bagui is Professor of Statistics. His areas of research are statistical classification and pattern recognition, bio-statistics, construction of designs, tolerance regions, and statistical computing. email: sbagui@uwf.edu. Dilip Ghosh is Professor and Head of the Department of Mathematics and Statistics. His areas of research are combinatorial designs, construction of designs and its analysis, optimal designs, biostatistics, graph theory, and statistical analysis of cancer data. email: ghosh_dkg@rediffmail.com. choice of appropriate design with a specified variance for the elementary (estimable) contrasts is very useful for inference purposes. Similar issues also arise in the design of industrial experiments for statistical quality control problems. There are situations where the value of $\sigma^{2}$ is known, but there is no procedure available for determining the parameters of a block design in order to compare treatment effects with a pre-determined variance for the elementary (estimable) contrasts under consideration.

Let there be $v$ treatments arranged in $b$ blocks, such that the $j$-th block contains $k_{j}$ experimental units and the $i$-th treatment appears $r_{i}$ times in the entire design, $i=1,2, \cdots, v ; j=1,2, \cdots, b$. The $v \times b$ matrix $N=\left(n_{i j}\right)$, where $n_{i j}$ is the number of times $i$-th treatment occurs in the $j$-th block, is the incidence matrix of the block design. If $K=\operatorname{diag}\left(k_{1}, \cdots, k_{b}\right) \quad$ and $R=\operatorname{diag}\left(r_{1}, \cdots, r_{v}\right)$, then the $C$-matrix of the design is defined as

$$
C=R-N K^{-1} N^{\prime}
$$


The following discussion is based on Gini (1986).

\section{Definition 1.1}

A contrast $\quad \sum_{j} C_{j} t_{j}=C^{\prime} t \quad$ with $C=\left(C_{1}, \cdots, C_{v}\right)^{\prime}$ in the treatment effects $t=\left(t_{1}, \cdots, t_{v}\right)^{\prime}$ is called an elementary contrast if $C$ has only two non-zero elements 1 and-1. It is often desirable to design experiments where all elementary contrasts $t_{i}-t_{j}, i \neq j$ are estimable.

\section{Definition 1.2}

A design where all elementary contrasts are estimable is called a connected design.

\section{Definition 1.3}

A connected design is said to be balanced if all elementary contrasts in the treatment effects can be estimated with the same precision.

\section{Definition 1.4}

A balanced incomplete block design (BIBD) is an arrangement of $v$ treatments in $b$ blocks each containing $k$ experimental units $(k<v)$ such that

(i) every treatment occurs at most once in each block,

(ii) every treatment occurs exactly in $r$ blocks

(iii) every pair of treatments occur together in exactly $\lambda$ blocks.

For any estimable contrast $\psi=\sum_{j} C_{j} t_{j}$ corresponding to a BIBD, the variance of its unbiased estimator $\hat{\psi}=\sum_{j} C_{j} \hat{t}_{j}$ is given by

$$
\operatorname{Var}(\hat{\psi})=(r \mathcal{E})^{-1} \sigma^{2} \sum_{j} C_{j}^{2}
$$

Where $\varepsilon=\frac{v(k-1)}{k(v-1)}$ is called the efficiency factor of BIBD. If it is a randomized block design (RBD), then

$$
\operatorname{Var}(\tilde{\psi})=\frac{\sigma^{2}}{r} \sum_{j} C_{j}^{2} .
$$

\section{Definition 1.5}

If the elements of the incidence matrix take only values 0 or 1 , then the incomplete block design is called a binary design.

Definition 1.6

An incomplete block design with $k_{1}=\cdots=k_{b}=k$ is called a proper design.

Some Properties of $C$-matrix

The $C$-matrix of block designs is very useful in the analysis and construction of various block designs. The following results on $C$-matrix are well known and more details can be seen from Dey(1986) and Raghavarao(1971).

1. For a balanced design all the non-zero characteristic roots of its $C$-matrix are equal.

2. For a balanced design each of the elementary contrasts is estimated with a variance $\frac{2 \sigma^{2}}{\theta}$, where $\theta$ is the non zero characteristic root of the $C$-matrix.

3. For a balanced design the C-matrix is of the form $C=\theta\left(I_{v}-v^{-1} J J^{\prime}\right)$ where $J=(1, \cdots, 1)^{\prime}$.

4. A block design is connected if the rank of its $C$-matrix is $v-1$.

5. In a connected design the diagonal elements of the $C$-matrix are all positive. Further, the principal minors of all orders $(1,2, \cdots, v-1)$ of $C$ are all positive.

6. A connected block design is variance balanced if its $C$-matrix has all its diagonal elements equal and all its off diagonal elements equal. That is, $C$ is given by $C=(a-b) I+b J J^{\prime}$ where $a$ and $b$ are some scalars. 
7. For an equireplicate, proper block design,

$$
C=r I-\frac{N N^{\prime}}{k}
$$

and if the design is binary,

$$
\operatorname{tr}(C)=v r-\frac{v r}{k}=v r-b .
$$

If it is variance balanced, then

$$
\operatorname{tr}(C)=\theta(v-1)
$$

If all the above conditions hold, then

$$
\theta=\frac{(v r-b)}{(v-1)}
$$

8. For any general incomplete block design with $\sum_{i j} n_{i j}=b k$,

$$
\operatorname{tr}(C)=b k-k^{-1} \sum_{i j} n_{i j}^{2}
$$

is maximized by the BIBD whenever it exists.

\section{Methodology}

A method for identifying optimal designs for comparing treatment effects of equireplicate, proper, variance balanced, and connected block designs is introduced. The procedure can be extended to other cases also.

\section{Determination of Optimal Designs}

Suppose that the process variability $\sigma^{2}$ is known. Then, following are the steps involved in determining the parameters of the corresponding design.

1. Decide upon the precision of the elementary (estimable) contrast to be estimated.
2. Choose an integer value for $\theta$ so that the variance of the elementary contrasts $\frac{2 \sigma^{2}}{\theta}$ will meet the specification in step 1.

3. Select an appropriate $C$-matrix of dimension $v \times v$ such that the non-zero characteristic root is $\theta$ which is determined in step 2. The value of $v$ is known from the objective of the underlying experiment.

The exploration of a C-matrix corresponding to a variance balanced and connected design is not tedious as it has the following format.

$$
\left(a_{i j}\right)=\left[\begin{array}{cccc}
a & b & \cdots & b \\
b & a & \cdots & b \\
\vdots & \vdots & \cdots & \vdots \\
b & b & \cdots & a
\end{array}\right]_{v x v}
$$

The characteristic roots of the above matrix are $a+(v-1) b$ and $(a-b)$ of multiplicity $v-1$.

4. Determine all possible combinations of $r$ and $b$ satisfying the condition $\theta(v-1)=v r-b$.

5. Since $\theta$ and $v$ are known quantities, one can consider those combinations of $r$ and $b$ satisfying the above condition. Among these combinations of $b$ and $r$, those values satisfying the condition $v r$ $=b k$, where $k$ must be an integer such that $k \leq v$ are selected. The existence of an incidence matrix corresponding to the resulting design must be verified. If it is a proper block design, then $N N^{\prime}$ will be such that

$$
N N^{\prime}=k(r I-C)
$$

If it is a BIBD, then

$$
N N^{\prime}=(r-\lambda) I+\lambda J J^{\prime}
$$


where $\lambda$ is such that $\lambda(v-1)=r(k-1)$. Hence, the following theorem.

\section{Theorem 2.1}

The above process will lead to a set of optimal designs, which include one RBD and BIBDs provided the latter should exist.

The resulting designs were called optimal designs because Kiefer (1958) proved that RBD and BIBD are $A, D, E$ and $L$ optimum. Further, Roy (1958) proved that in the case of proper binary incomplete block designs, a most efficient design if it exists, is necessarily a BIBD.

\section{Corollary 2.1}

Corresponding to a given $C$-matrix there can be more than one design with the same variance for all the elementary contrasts.

\section{Theorem 2.2}

If $\theta$ is the non-zero characteristic root of the $C$-matrix corresponding to an RBD, then $\theta=b=r$.

Proof. The $C$-matrix corresponding to an RBD satisfies the condition

$$
C=r I-\frac{N N^{\prime}}{k}
$$

and hence

$$
\operatorname{tr}(C)=v r-\frac{v r}{k}=v r-b
$$

Because $v r=b k$ it can be rewritten as

$$
\operatorname{tr}(C)=b(k-1)
$$

Further the design is variance balanced and hence all the non-zero characteristic roots of $C$ matrix are equal to $\theta$. Hence

$$
\operatorname{tr}(C)=\theta(v-1)
$$

From (2) and (3) it follows that

$$
\theta=\frac{b(k-1)}{(v-1)}
$$

Now $v$ and $k$ are equal in the case of RBD and hence $\theta=b$. Because $v r=b k, v=k$ implies $r=b$.

The above result is justified because the variance of each elementary contrast of a RBD is $\frac{2 \sigma^{2}}{r}$. Therefore, an RBD with a suitable $r$ will have a required precision for its estimable contrasts. Table 2.1 gives the manner in which $\theta$ changes with respect to the other parameters of an RBD having 5 treatments.

Table 2.1

\begin{tabular}{|c|c|c|c|c|c|c|c|}
\hline$r$ & 2 & 3 & 4 & 5 & 6 & 7 & 8 \\
\hline$b$ & 2 & 3 & 4 & 5 & 6 & 7 & 8 \\
\hline$k$ & 5 & 5 & 5 & 5 & 5 & 5 & 5 \\
\hline$\theta$ & 2 & 3 & 4 & 5 & 6 & 7 & 8 \\
\hline
\end{tabular}

Suppose one wishes to compare the effects of $v$ treatments with the variance of the elementary contrast being $\frac{2 \sigma^{2}}{\theta}$, where $\theta$ is the non-zero characteristic root of the $C$-matrix of a variance balanced design. The following example illustrates the method of determining the values of $b, r$, and $k$ corresponding to an optimal design.

\section{Example}

Let $v=5$ and $\theta=5$. Then the $C$-matrix of a block design with non-zero characteristic root as 5 can be given as

$$
C=\left[\begin{array}{ccccc}
4 & -1 & -1 & -1 & -1 \\
-1 & 4 & -1 & -1 & -1 \\
-1 & -1 & 4 & -1 & -1 \\
-1 & -1 & -1 & -1 & 4
\end{array}\right]
$$

values of $b, r$, and $k$ are to be determined such that there exists integers satisfying the 
conditions $v r=b k$ and $\theta(v-1)=v r-b$. One can easily verify that the following three designs which satisfy all the requirements given in steps 1 to 4 .

1. RBD with parameters $b=r=k=5$

2. BIBD with parameters $b=10, r=$ $6, k=3, \lambda=3$.

3. BIBD with parameters $b=10, r=$ $8, k=2, \lambda=2$.

More examples are presented in table 2.2.

From the above examples it can be seen that corresponding to a positive integer $\theta$ there always exists an RBD described in Theorem 2 but BIBDs need not exist for a positive integer $\theta$. Note that if BIBDs exist then the parameters will satisfy the relation $\theta=\frac{\lambda v}{k}$.

\section{References}

Dey, A. (1986). Theory of block designs. Wiley Eastern Limited.

Giri, N. (1986). Analysis of variance. New Delhi: South Asian Publishers.

Kiefer, J. (1958). On the nonrandomized optimality and randomized nonoptimality of symmetrical designs. The Annals of Mathematical Statistics 29, 675-699.

Raghavarao, D. (1971). Constructions and combinatorial problems in design of experiments. New York, N.Y.: Wiley.

Roy, J. (1958). On the efficiency factor of block designs. Sankhya 19, 181-188.

Table 2.2

\begin{tabular}{|c|c|c|c|c|}
\hline$v$ & $\theta$ & $C$-matrix of order $v$ & RBD Parameters & RBD Parameters \\
\hline 3 & 3 & $c_{i i}=2, c_{i j}=-1, i \neq j$ & $r=b=k=3$ & $b=6, r=4, k=2, \lambda=2$ \\
\hline 5 & 10 & $c_{i j}=8, c_{i j}=-2, i \neq j$ & $r=b=10, k=5$ & $b=20, r=12, k=3, \lambda=6$ \\
\hline 6 & 6 & $c_{i i}=5, c_{i j}=-1, i \neq j$ & $r=b=k=6$ & $b=30, r=10, k=2, \lambda=2$ \\
\hline 6 & 12 & $c_{i i}=10, c_{i j}=-2, i \neq j$ & $r=b=12, k=6$ & $b=24, r=8, k=3, \lambda=2$ \\
\hline 9 & 6 & $c_{i i}=\frac{16}{3}, c_{i j}=-\frac{2}{3}, i \neq j$ & $r=b=6, k=9$ & $b=24, r=8, k=3, \lambda=2$ \\
\hline 7 & 3 & $c_{i i}=\frac{5}{2}, c_{i j}=-\frac{1}{2}, i \neq j$ & $r=b=3, k=7$ & Does not exist \\
\hline 8 & 16 & $c_{i i}=14, c_{i j}=-2, i \neq j$ & $r=b=16, k=8$ & Does not exist \\
\hline
\end{tabular}

